\title{
Molecular Characterization of Binding Loop E in the Nematode Cys-Loop GABA Receptor ${ }^{\text {\ }}$
}

\author{
Ariel Kwaka, Mohammad Hassan Khatami, Joshua Foster, Everett Cochrane, \\ Sarah A. Habibi, Hendrick W. de Haan, and Sean G. Forrester \\ Faculty of Science, University of Ontario Institute of Technology, Oshawa, Ontario, Canada
}

Received May 2, 2018; accepted August 30, 2018

\begin{abstract}
Nematodes exhibit a vast array of cys-loop ligand-gated ion channels with unique pharmacologic characteristics. However, many of the structural components that govern the binding of various ligands are unknown. The nematode cys-loop GABA receptor uncoordinated 49 (UNC-49) is an important receptor found at neuromuscular junctions that plays an important role in the sinusoidal movement of worms. The unique pharmacologic features of this receptor suggest that there are structural differences in the agonist binding site when compared with mammalian receptors. In this study, we examined each amino acid in one of the main agonist binding loops (loop E) via the substituted cysteine accessibility method (SCAM) and analyzed the interaction of various residues by molecular dynamic
\end{abstract}

simulations. We found that of the 18 loop $\mathrm{E}$ mutants analyzed, $\mathrm{H} 142 \mathrm{C}, \mathrm{R} 147 \mathrm{C}$, and S157C had significant changes in GABA $\mathrm{EC}_{50}$ and were accessible to modification by a methanethiosulfonate reagent (MTSET) resulting in a change in $I_{\mathrm{GABA}}$. In addition, the residue $\mathrm{H} 142$, which is unique to nematode UNC49 GABA receptors, appears to play a negative role in GABA sensitivity as its mutation to cysteine increased sensitivity to GABA and caused the UNC-49 receptor partial agonist 5-aminovaleric acid (DAVA) to behave as a full agonist. Overall, this study has revealed potential differences in the agonist binding pocket between nematode UNC-49 and mammalian GABA receptors that could be exploited in the design of novel anthelmintics.

\section{Introduction}

Nematodes such as the model free-living nematode Caenorhabditis elegans and parasitic nematodes such as Haemonchus contortus contain a vast array of cys-loop ligand-gated chloride channels that respond to a variety of neurotransmitters including serotonin, tyramine, dopamine, GABA, and glutamate (Jones and Sattelle, 2008; Bartos et al., 2009; Laing et al., 2013). In addition, nematodes exhibit a unique class of acetylcholine-gated chloride channels (Putrenko et al., 2005) and GABA-gated cation channels (Beg and Jorgensen, 2003). However, like vertebrates, nematodes also exhibit anion selective receptors for GABA.

The nematode cys-loop GABA receptor that is the most characterized pharmacologically and functionally is the uncoordinated 49 (UNC-49) receptor (Bamber et al., 1999; Siddiqui et al., 2010). This receptor is expressed at the neuromuscular junctions of nematodes and contributes to the worm's sinusoidal movement (Bamber et al., 2005). With regard to amino acid sequence, the UNC-49 receptor does not appear to be

Research was funded by the Natural Science and Engineering Council of Canada (NSERC) and the Canadian Foundation for Innovation (CFI) to S.G.F. PDB File: Hco-UNC-49B homodimer.

https://doi.org/10.1124/mol.118.112821.

S This article has supplemental material available at molpharm. aspetjournals.org. analogous to any mammalian GABA receptor, and it appears to exhibit unique pharmacologic features. One significant pharmacologic difference is the low sensitivity of the classic GABA receptor antagonist bicuculline (Bamber et al., 2003), which is a characteristic shared among invertebrate GABA receptors (Hosie et al., 1997). Differences have also been observed between the agonist profile of the UNC-49 receptor from $H$. contortus (Hco-UNC-49) and the mammalian receptors. Most notable is the insensitivity of the nematode receptor to sulphonated compounds such as taurine and $\mathrm{P} 4 \mathrm{~S}$, which have been shown

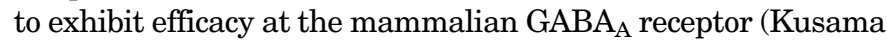
et al., 1993; Woodward et al., 1993; del Olmo et al., 2000; Kaji et al., 2015).

The unique pharmacologic features of nematode cys-loop GABA receptors suggest that there are structural differences in the agonist binding pocket. However, compared with their mammalian counterparts, little is known about the structural elements that govern agonist binding to nematode GABA receptors.

The binding site of cys-loop receptors is made up of six discontinuous loops, designated by the letters $\mathrm{A}$ to $\mathrm{F}$. The number of loops and their general position appear conserved across phyla (Brejc et al., 2001; Hibbs and Gouaux, 2011). Loops $\mathrm{A}$ to $\mathrm{C}$ are found within the primary subunit, and loops $\mathrm{D}$ to $\mathrm{F}$ are in the adjacent subunit. The role of various binding

ABBREVIATIONS: cRNA, copy RNA; DAVA, 5-aminovaleric acid; MD, molecular dynamics; MS-222, 3-aminobenzoic acid ethyl ester methane sulphonate salt; MTSET, 2-(trimethylammonium)ethyl methanethiosulfonate; ND96 buffer, [96 mM NaCl, $2 \mathrm{mM} \mathrm{KCl} 1.8 \mathrm{mM} \mathrm{CaCl}_{2}, 1 \mathrm{mM} \mathrm{MgCl}$, 5 mM HEPES]; PS, pregnenolone sulfate; RDL, resistance to dieldrin; SCAM, substituted cysteine accessibility method; UNC, uncoordinated; WT, wild type. 
loop residues in the function of mammalian GABA receptors have been examined through site-directed mutagenesis and the substituted cysteine accessibility method (SCAM).

SCAM analysis involves changing the predicted binding site residues to cysteines via site-directed mutagenesis. If the introduced cysteine is modified by a reducing agent such as 2-(trimethylammonium)ethyl methanethiosulfonate (MTSET) (see Fig. 1), GABA binding should be reduced if that cysteine resides is in the binding pocket. Binding pocket residues are confirmed through the use of agonists and antagonists, which slow the rate of modification of the introduced cysteines by reducing agents (Kloda and Czajkowski, 2007). However, it is important to note that modification of cysteines by molecules such as MTSET could have more complex effects on receptor activation, so the results should be interpreted with caution.

The detailed knowledge that we now have about the structure of the GABA binding pocket in mammalian GABA receptors has provided an opportunity for comparative analyses on the GABA receptors of more primitive organisms such as nematodes. Several residues in loops A to D have previously been examined in the Hco-UNC-49 receptor through sitedirected mutagenesis, which revealed functional similarities when compared with mammalian GABA receptors (Accardi and Forrester, 2011). Binding loop E, on the other hand, is made up of 18 residues; the residues that play key roles in ligand binding have been mapped in both the $\mathrm{GABA}_{\mathrm{A}} \alpha 1$ and the GABAc $\rho 1$ subunits via SCAM analysis (Sedelnikova et al., 2005; Kloda and Czajkowski, 2007). We aligned loop E from these two GABA receptor classes with the Hco-UNC-49 receptor, revealing 12 positions that are not completely conserved (Fig. 2).

Interestingly, residues such as $\mathrm{H} 142$ and F145 appear to be conserved among nematode UNC-49-like receptors. We wondered whether the unique residues present in loop $\mathrm{E}$ of nematode GABA receptors have any functional significance when compared with published reports on mammalian receptors and if they could possibly explain some of the pharmacologic characteristics observed. We conducted an examination of each residue in loop $\mathrm{E}$ from the $H$. contortus UNC-49 receptor using site-directed mutagenesis and SCAM, two methods that have been used to characterize the mammalian GABA binding site, to gain some insight into differences between mammalian and nematode GABA receptors. Of the residues we examined experimentally and via molecular dynamic simulations, some appeared to have similarity in function with mammalian receptors, but others could have some functional differences.

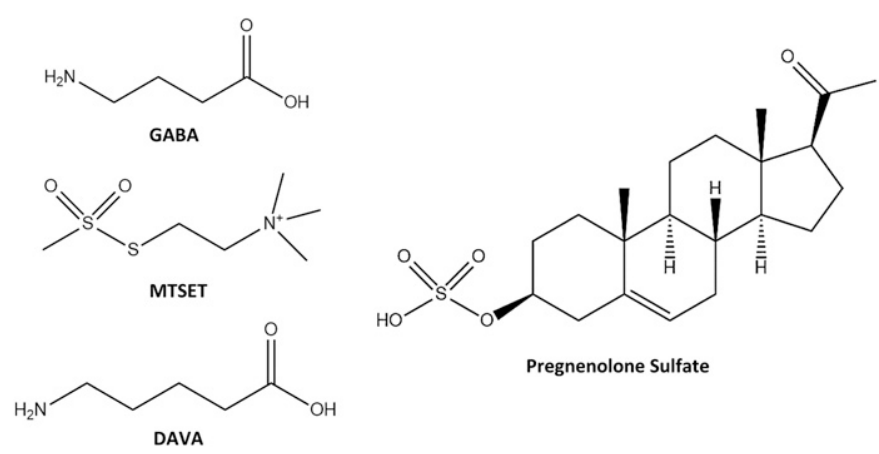

Fig. 1. Chemical structures of GABA, MTSET, DAVA, and PS.

\section{Materials and Methods}

Site-Directed Mutagenesis-Primer Design. Amino acid alignments of Hco-UNC-49B and other GABA receptor subunits in the ligandgated chloride channel (LGCC) family, were used to determine the location of loop $\mathrm{E}$ within the $H$. contortus sequence (Fig. 2). The primers were designed using Stratagene's web-based QuikChange Primer Design program (www.stratagene.com/sdmdesigner/default.aspx) to create 18 Hco-unc-49b (GenBank Accession EU939734.1) mutants: H142C, N143C, S144C, F145C, L146C, R147C, I148C, D149C, S150C, D151C, G152C, T153C, V154C, Y155C, T156C, S157C, Q158C, and R159C. Each pair of mutagenic primers was designed to change each amino acid residue within loop $\mathrm{E}$ into cysteine residues by way of nucleotide introduction, resulting in singly mutated cysteine mutants.

Site-Directed Mutagenesis of Hco-unc-49b. Previous work in our laboratory found that the binding site for GABA in the Hco-UNC49BC channel was at the interface of two adjacent UNC-49B subunits. However, the exact stoichiometry of the heteromeric channel is unknown (Accardi and Forrester, 2011). The template used in the site-directed mutagenesis of $\mathrm{Hco}-u n c-49 b$ was previously subcloned into a pT7Ts transcription vector, in which Xenopus laevis $\beta$-globin untranslated DNA was incorporated into the $5^{\prime}$ and $3^{\prime}$ ends of the HcoUNC-49B subunit cDNA (Siddiqui et al., 2010).

A naturally occurring cysteine residue located at position 224 of the template amino acid sequence was removed through site-directed mutagenesis to prevent interference with the results obtained from the introduced cysteine residues. With no alteration in function (Fig. 3;

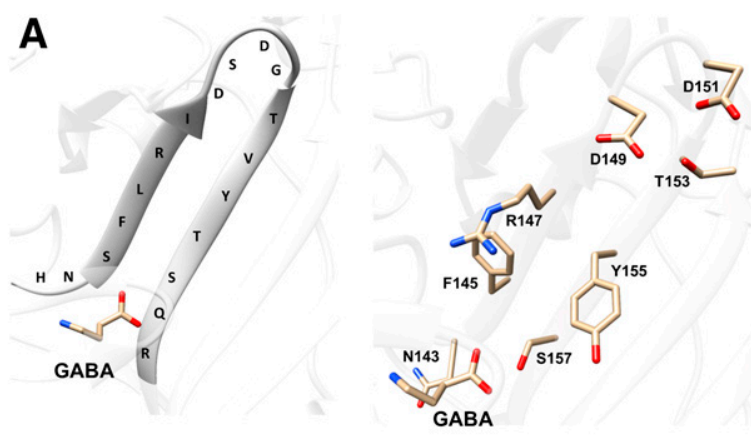

B

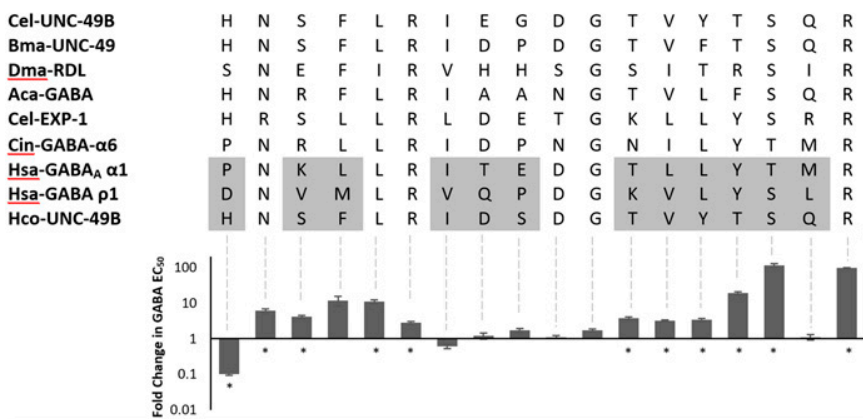

Fig. 2. (A) Model of Hco-UNC-49B homodimer with a docked GABA molecule, highlighting loop $\mathrm{E}$ residue positions (left) and the residues that face the binding pocket (right) based on the GluCl crystal structure (PDB 3RIF). (B) Sequence alignment of Hco-UNC-49B loop E residues with the loop E residues from other Cys-loop receptors (Caenorhabditis elegans UNC49, Brugia malayi UNC-49, Drosophila melanogaster RDL, Aplysia californica GABA receptor $\alpha$-like subunit, Caenorhabditis elegans EXP-1, Ciona intestinalis GABA $\alpha 6$-like subunit, human $\mathrm{GABA}_{\mathrm{A}}$, and human $\mathrm{GABA}_{\mathrm{C}}$ ). Shaded area indicates positions not conserved between Hco-UNC-49B, Hsa$\mathrm{GABA}_{\mathrm{A}} \alpha 1$, and Hsa-GABA $\rho 1$. Bar graph below the alignment demonstrates the fold change in GABA EC Fo $_{50}$ of HNC-UNC-49BC observed when each HcoUNC-49B loop E amino acid was replaced with a cysteine via site-directed mutagenesis. Each bar represents an average fold change $(n \geq 6) \pm$ S.E. The associated $\mathrm{EC}_{50}$ values that were statistically different $(P>0.001)$ from C224A are indicated by an asterisk(*). PBD File: Hco-UNC-49. 
A

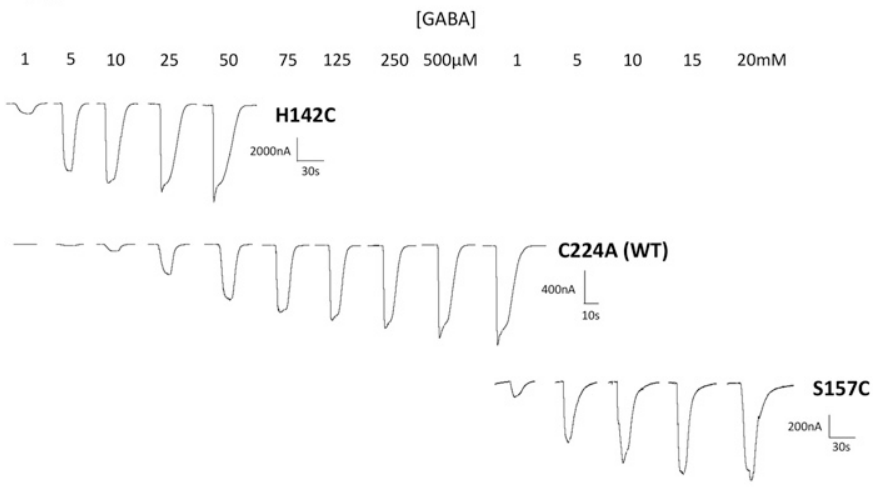

B

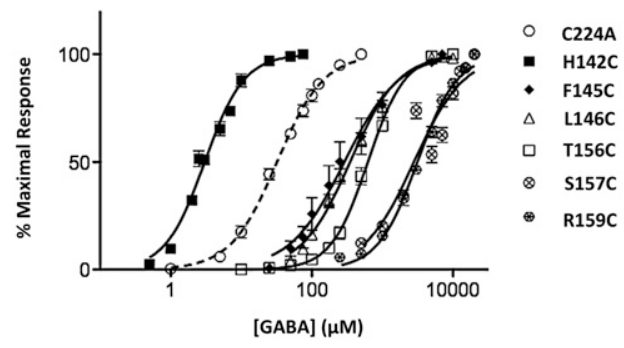

Fig. 3. (A) Representative electrophysiological tracings of Hco-UNC49BC with mutated Hco-UNC-49B subunits H142C, C224A (cysteine-less mutant used as template for creation of other mutants), and S157C. (B) Dose-response curve of Hco-UNC-49B mutants showing differences in GABA sensitivity, with normalized currents. Each point represents the mean with bars of S.E. $(n>6)$.

Table 1), the cysteine-less mutant (C224A) was used as template and a baseline from which all data obtained could be compared throughout.

All site-directed mutagenesis was conducted using the QuikChange Site-Directed Mutagenesis Kit (Stratagene, La Jolla, CA) according to the manufacturer's instructions. Confirmation that the correct mutation was present was verified using DNA sequencing (Génome Québec, Montreal, Canada).

cRNA Preparation-In Vitro Transcription. Plasmid constructs containing either the mutated Hco-unc-49b and wild-type (WT) Hcounc-49c (GenBank Accession EU049602.1) were linearized and used as a template $(0.4-1.0 \mu \mathrm{g})$ to create capped Hco-unc-49 copy RNA (cRNA). The cRNA was made using the mMessage mMachine in vitro transcription reaction and the T7 RNA polymerase provided within the transcription kit (Ambion, Austin, TX). The cRNA was precipitated using lithium chloride and was brought to a concentration of $0.5 \mathrm{ng} / \mathrm{l}$ upon resuspension in nuclease free water. Each in vitro transcription reaction yielded approximately 10-25 $\mu \mathrm{g}$ of cRNA.

Expression of Hco-UNC-49BC in Xenopus laevis Oocytes. Oocytes were obtained surgically from female Xenopus laevis (Nasco, Fort Atkinson, WI), which were housed in temperature-controlled rooms at the University of Ontario Institute of Technology (UOIT) according to the methodology outlined in Abdelmassih et al. (2017) and adhering to the guidelines of the UOIT Animal Care Committee and the Canadian Council of Animal Care. The frogs were anesthetized with $0.15 \% 3$-aminobenzoic acid ethyl ester methane sulphonate salt (MS-222; Sigma-Aldrich, Oakville, ON, Canada). Lobes of the ovary were extracted, split into smaller pieces of 10-20 oocytes, and defolliculated in a treatment of $2 \mathrm{mg} / \mathrm{ml}$ collagenase-II (SigmaAldrich) and OR2 solution ( $82 \mathrm{mM} \mathrm{NaCl}, 2 \mathrm{mM} \mathrm{KCl}, 1 \mathrm{mM} \mathrm{MgCl}_{2}$, $5 \mathrm{mM}$ HEPES, $\mathrm{pH}$ 7.5) for 2 hours with gentle rocking at room temperature. After defolliculation, the oocytes were stored at $20^{\circ} \mathrm{C}$ in ND96 buffer (96 mM NaCl, $2 \mathrm{mM} \mathrm{KCl,} 1.8 \mathrm{mM} \mathrm{CaCl}_{2}, 1 \mathrm{mM} \mathrm{MgCl}_{2}$,
TABLE 1

Comparison of GABA $\mathrm{EC}_{50}$ and Hill slope values of Hco-UNC-49B mutants assembled as heteromeric Hco-UNC-49BC receptors

Fold change values indicate change in $\mathrm{GABA}_{\mathrm{EC}_{50}}$ of each mutant normalized to C224A mutant (cysteine-less mutant with wild-type responses, used as template for creation of other loop $\mathrm{E}$ mutants).

\begin{tabular}{lrrr}
\hline Mutant & $\begin{array}{c}\mathrm{EC}_{50} \pm \text { S.E. }(\mu \mathrm{M}) \\
\text { (Hill Coefficient } \pm \text { S.E. })\end{array}$ & $n$ & $\begin{array}{c}\text { Fold } \\
\text { Change }\end{array}$ \\
\hline C224A & $32.56 \pm 2.29(1.37 \pm 0.11)$ & 7 & 1 \\
H142C & $2.70 \pm 0.22(1.44 \pm 0.07)^{a}$ & 7 & 0.1 \\
N143C & $199.69 \pm 22.86(1.33 \pm 0.11)^{a}$ & 7 & 6.1 \\
S144C & $133.76 \pm 11.96(1.31 \pm 0.07)^{a}$ & 7 & 4.1 \\
F145C & $383.53 \pm 114.30(1.40 \pm 0.04)$ & 7 & 11.8 \\
L146C & $361.39 \pm 42.59(1.30 \pm 0.04)^{a}$ & 7 & 11.1 \\
R147C & $90.32 \pm 6.54(1.82 \pm 0.16)^{a}$ & 8 & 2.8 \\
I148C & $19.37 \pm 2.62(1.53 \pm 0.13)$ & 8 & 0.6 \\
D149C & $39.79 \pm 7.11(2.15 \pm 0.27)$ & 7 & 1.2 \\
S150C & $54.39 \pm 6.95(1.78 \pm 0.13)$ & 7 & 1.7 \\
D151C & $36.11 \pm 3.25(1.43 \pm 0.08)$ & 7 & 1.1 \\
G152C & $54.91 \pm 5.01(1.48 \pm 0.06)$ & 7 & 1.7 \\
T153C & $122.21 \pm 10.01(1.71 \pm 0.08)^{a}$ & 6 & 3.8 \\
V154C & $104.05 \pm 5.10(1.51 \pm 0.14)^{a}$ & 7 & 3.2 \\
Y155C & $111.49 \pm 10.68(1.64 \pm 0.09)^{a}$ & 7 & 3.4 \\
T156C & $628.48 \pm 50.61(1.71 \pm 0.06)^{a}$ & 6 & 19.3 \\
S157C & $3683.50 \pm 413.66(1.43 \pm 0.09)^{a}$ & 8 & 113.1 \\
Q158C & $35.44 \pm 6.63(1.44 \pm 0.10)$ & 7 & 1.1 \\
R159C & $3102.33 \pm 130.43(1.53 \pm 0.03)^{a}$ & 6 & 95.3 \\
\hline
\end{tabular}

${ }^{a}$ Values statistically significantly different from C224A $(P<0.001)$.

$5 \mathrm{mM}$ HEPES, pH 7.5) supplemented with $100 \mu \mathrm{g} / \mathrm{ml}$ gentamycin and $0.275 \mu \mathrm{g} / \mathrm{ml}$ pyruvic acid (Sigma-Aldrich). Supplemented ND96 solution was replaced twice every 24 -hour period.

Cytoplasmic injections of cRNA were performed on stage $\mathrm{V}$ and VI oocytes using a Drummond Nanoject II (Drummond Scientific Company, Broomhall, PA) assisted by micromanipulators (World Precision Instruments, Sarasota, FL). Each oocyte was injected with $50 \mathrm{nl}$ of a mixture of mutated Hco-unc-49b and WT Hco-unc-49c (equal amounts of $0.5 \mathrm{ng} / \mathrm{nl}$ cRNA of each). Electrophysiological recordings were taken after receptor expression, approximately 2 to 5 days after the cRNA injection.

Compounds Tested and Their Preparation. MTSET was obtained from Toronto Research Chemicals (Toronto, ON, Canada). GABA, 5-aminovaleric acid (DAVA), and pregnenolone sulfate (PS) were obtained from Sigma-Aldrich. All compounds were initially dissolved in ND96 except for PS, which was dissolved in dimethylsulfoxide for the preparation of a stock solution. Compounds were diluted in ND96 as working solutions. The structures of the compounds are found in Fig. 1.

Two-Electrode Voltage Clamp Electrophysiology. Using an Axoclamp900A voltage clamp (Molecular Devices, Sunnyvale, CA), two-electrode voltage clamp electrophysiology was used to observe and record the channel activity of the Hco-UNC-49B/C channel. Glass electrodes were pulled from borosilicate capillaries using a P-97 Flaming/Brown micropipette puller (Sutter Instruments, Novato, $\mathrm{CA}$ ), and filled with $3 \mathrm{M} \mathrm{KCl} \mathrm{(1-5} \mathrm{M} \Omega$ resistance). Each of the two electrodes were connected to Axon Instrument Headstages (Molecular Devices) using $\mathrm{Ag} \mid \mathrm{AgCl}$ wires.

Oocytes were held at a membrane voltage at $-60 \mathrm{mV}$ throughout the experiment. Compounds were perfused over the oocytes (at $8 \mathrm{ml} / \mathrm{min}$ ) through a gravitational flow system into an RC-1Z perfusion chamber (Warner Instruments, Holliston, MA). ND96 buffer was used to dilute the various compounds, in addition to being used as a wash solution in between drug applications. Recordings and associated data were obtained using Axon Instruments Digidata 1440, Clampex 10.2, and were analyzed via Clampfit 10.2 (Molecular Devices).

The $\mathrm{EC}_{50}$ was determined for each mutant by perfusing increasing concentrations of GABA (or other agonists) and recording the current observed which included the concentration of agonist that produced maximal current.

For experiments using the negative allosteric modulator of GABA receptors, PS, oocytes expressing mutant or WT Hco-UNC-49BC 
receptors were first exposed to $\mathrm{EC}_{50}$ concentrations of GABA to ensure channel functionality. Oocytes were then exposed to a coapplication of an increasing range (5-500 $\mu \mathrm{M})$ of PS concentrations mixed with the corresponding $\mathrm{EC}_{50} \mathrm{GABA}$ concentration. Changes in current were recorded and inhibitory dose-response curves were generated to determine the $\mathrm{IC}_{50}$ value for the mutant being tested.

Substituted Cysteine Accessibility Method. SCAM was used to characterize all Hco-UNC-49B mutants as well as the cysteine-less mutant $(\mathrm{C} 224 \mathrm{~A})$ using the reducing agent MTSET. During testing, an oocyte was washed and hit with $\mathrm{EC}_{20-50}$ concentrations of GABA in 5-minute intervals until the response stabilized within $10 \%$. Once stabilized, MTSET ( $1 \mathrm{mM}$ for 1 minute) was perfused over the oocyte, followed by 5 minutes of washing with ND96 solution. The $\mathrm{EC}_{20-50}$ concentrations of GABA for the mutant being tested were reapplied, and currents were recorded (Sedelnikova et al., 2005; Kloda and Czajkowski, 2007).

Statistical Analysis. Dose-response curves were generated by Prism 5.0 (GraphPad Software, San Diego, CA) using the following equation in the set-up log (agonist) versus normalized responsevariable slope:

$$
I_{\max }=\frac{1}{1+\left(\frac{\mathrm{EC}_{50}}{[D]}\right)^{h}}
$$

where $I_{\max }$ is the maximal response, $[D]$ is the concentration of agonist, $\mathrm{EC}_{50}$ is the concentration of agonist that is required to produce half-maximal current, and $h$ is the Hill coefficient. Responses used to produce dose-response curves were normalized as a percentage of the maximal current produced by the oocytes individual maximal response to GABA.

The effect of MTSET modification was calculated using the following formula:

$$
\left(\frac{I_{\mathrm{GABA}-\text { post }}}{I_{\mathrm{GABA}-\mathrm{pre}}}\right)-1
$$

where $I_{\text {GABA-post }}$ is the current produced from GABA activation of the receptor after the application of MTSET, and $I_{\mathrm{GABA}-p r e}$ is the current produced from GABA activation before MTSET was applied (Kloda and Czajkowski, 2007).

The $\mathrm{EC}_{50}$ values, $h$ values, and S.E. were determined using Prism 5.0 from a minimum of five oocytes from two different $X$. laevis. Statistical significance was derived from Student's $t$ test using the Bonferroni correction or a one-way analysis of variance with Dunnett's multiple comparison test. $P<0.001$ was considered statistically significant.

Homology Modeling. The C. elegans glutamate-gated chloride channel crystal structure (PDB 3RIF; Hibbs and Gouaux, 2011) was used as a template in MODELER 9.14 (Sali and Blundell, 1993) for the generation of a Hco-UNC-49B extracellular domain homodimer (Data Supplement PDB File). The most energetically favorable models were determined based on their DOPE score and PROCHECK Ramachandran plot analysis, as described in Kaji et al. (2015).

Computational Agonist Docking. The energetically reduced zwitterion form of GABA was obtained from the Zinc database (http://zinc.docking.org; Irwin et al., 2012). The GABA molecule was prepared for docking using AutoDock Tools (Morris et al., 2009) and was docked using AutoDock Vina (Trott and Olson, 2010). The center of the $30 \times 30 \times 30 \AA$ search box located in the aromatic box of the agonist binding site was used for agonist docking. A maximum of 50 binding models, all within a range of $5 \mathrm{kcal} \mathrm{mol}^{-1}$ from the best scoring pose, were generated (Kaji et al., 2015).

Molecular Dynamics Simulations. The homology model of the protein we generated, containing the docked GABA molecule in the binding area, was used as the initial structure for the molecular dynamics (MD) simulations. The GROMACS 2016.4 software package (www.gromacs.org), employing the CHARMM36 force field (Best et al., 2012), was used to run the simulations. The CHARMM General Force
Field (CGenFF) program, which is an automated method to provide parameters and charges by analogy (Vanommeslaeghe et al., 2010, 2012; Vanommeslaeghe and MacKerell, 2012; Yu et al., 2012; CGenFF interface at https://cgenff.paramchem.org), was used to acquire the CHARMM force field parameters for the GABA molecule. The simulation box was solvated with $\sim 21,000$ tip3p water molecules, along with $\mathrm{Cl}^{-}$counter ions to neutralize the total charge of the system.

To carry out the MD simulation, after a brief energy minimization, a 2 nanosecond equilibration simulation was performed under the NVT (number of particle, volume of the system, and temperature are constant) conditions at $300 \mathrm{~K}$ and a time step of 2 femtoseconds. The positions of the protein and the ligand were restrained to prevent any structural changes during the equilibration process.

In the production run, under the NPT (number of particle, pressure of the system, and temperature are constant) conditions, the system was kept at $300 \mathrm{~K}$ and $1 \mathrm{~atm}$ with a time step of 2 femtoseconds for 500 nanoseconds. The Parrinello-Rahman isotropic pressure coupling (with $\tau p=5$ picoseconds and compressibility $4.5 \times 10^{-5} \mathrm{bar}^{-1}$ ) and the Nose-Hoover thermostat (with a time constant of 0.1 picoseconds) were employed for these production runs. Long-range electrostatics were calculated with the particle-mesh Ewald method. The trajectory was printed every 10 picoseconds.

The gromacs utilities, such as gmx-mpi mindist, gmx_mpi gyrate, and gmx_mpi rms, as well as the H-bond calculator plugin of VMD (Visual Molecular Dynamics) (Humphrey et al., 1996; http://www.ks. uiuc.edu/Research/vmd/), were used to calculate the results. The default values for distance $(d)$ and angle $(\theta)\left(d<3.0 \AA\right.$ and $\left.\theta<20^{\circ}\right)$ are used to determine the H-bonds. Ionic bonds between oppositely charged residues were determined at $d<3.0 \AA$.

\section{Results}

Characterization of Loop E Cysteine Mutants in HcoUNC-49BC. The importance of loop E amino acid residues in the Hco-UNC49 receptor was first assessed by determining the impact of each cysteine mutation on the GABA activation of the channel. Each loop E mutant was exposed to increasing concentrations of GABA to determine the $\mathrm{EC}_{50}$ values. Upon recording, good expressions of all receptors with mutated subunits were observed, all receptors were functional, and clean tracings were obtained (Fig. 3A). The cysteine-less mutant C224A was an appropriate control for this study as the GABA EC G0 $_{50}(33 \mu \mathrm{M})$ was similar to WT ( $40 \mu \mathrm{M}$; Siddiqui et al., 2010), and this mutant was not sensitive to MTSET treatment.

Of the 18 mutants tested, 12 showed a substantial shift in GABA $\mathrm{EC}_{50}$ compared with the C224A cysteine-less mutant from which they were derived (representative dose-response curves are seen in Fig. 3B). Of the mutants that displayed a different $\mathrm{EC}_{50}$ from $\mathrm{C} 224 \mathrm{~A}$ (33 $\mu \mathrm{M} \pm 2$ ), $\mathrm{H} 142 \mathrm{C}$ showed an increase in GABA sensitivity $(2.7 \mu \mathrm{M} \pm 0.2)$. The remaining 11 mutants (N143C, S144C, F145C, L146C, R147C, T153C, V154C, Y155C, T156C, S157C, and R159C) had a decrease in GABA sensitivity, with $\mathrm{S} 157 \mathrm{C}$ and R159C showing the greatest decrease $(3683.50 \mu \mathrm{M} \pm 413.66$ and $3102.33 \mu \mathrm{M} \pm 130.43$, respectively). Finally, six of the cysteine mutants, I148C, D149C, S150C, D151C, G152C, and Q158C, showed minimal change in $\mathrm{GABA} \mathrm{EC}_{50}$. The $\mathrm{EC}_{50}$ values that were statistically significantly different from C224A $(P>0.001)$ are shown in Table 1.

Determination of Cysteine Accessibility. SCAM was performed on all loop E Hco-UNC-49B mutants to determine which were accessible to MTSET modification and the impact of modification on channel function. Overall, we found that 
most of the cysteine mutants were either not influenced or positively influenced by MTSET treatment. Specifically, out of the 18 mutants tested, only H142C, R147C, and S157C were significantly $(P<0.001)$ affected by MTSET modification (Fig. 4). Furthermore, only S157C had a decrease in the percentage change in $I_{\mathrm{GABA}}(-49.8 \% \pm 7.8 \%)$. Conversely, $\mathrm{H} 142 \mathrm{C}$ and $\mathrm{R} 147 \mathrm{C}$ all showed an increase in the percentage change in $I_{\mathrm{GABA}}(88 \% \pm 4 \%$ and $52.5 \% \pm 17 \%$, respectively).

Pharmacologic Characterization of H142C Mutant. Upon discovering significant hypersensitivity to GABA in H142C, we investigated two other compounds: DAVA and PS. Dose-response curves were created for both compounds (Fig. 5, A and C) with respect to the H142C mutant as well as the cysteine-less $\mathrm{C} 224 \mathrm{~A}$ baseline mutant. In addition, DAVA behaved as a partial agonist at the $\mathrm{C} 224 \mathrm{~A}$ receptor $\left(I_{\max } 21.2 \% \pm 2.7 \%\right)$, but as a full agonist at the $\mathrm{H} 142 \mathrm{C}$ receptor $\left(I_{\max } 86.5 \% \pm 2.8 \%\right.$; Table 2$)$. On the other hand, no significant difference was observed in the $\mathrm{IC}_{50}$ of $\mathrm{PS}$ between the $\mathrm{C} 224 \mathrm{~A}$ and $\mathrm{H} 142 \mathrm{C}$ receptors (Fig. 5; Table 2). For the $\mathrm{C} 224 \mathrm{~A}$ receptor, the $\mathrm{IC}_{50}$ for $\mathrm{PS}$ was $47 \mu \mathrm{M}$ compared with $70 \mu \mathrm{M}$ for $\mathrm{H} 142 \mathrm{C}$.

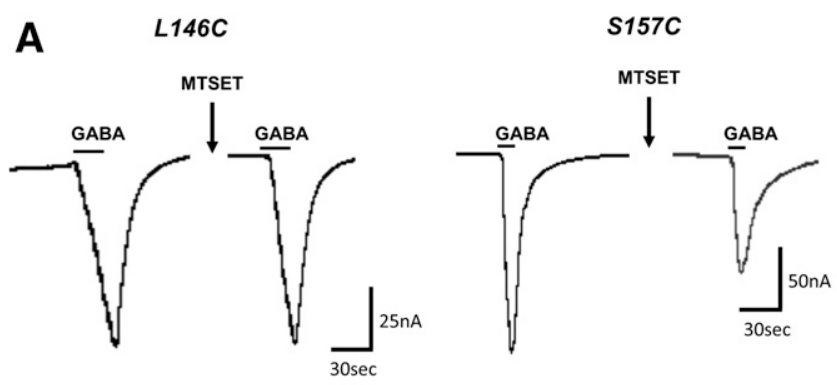

B

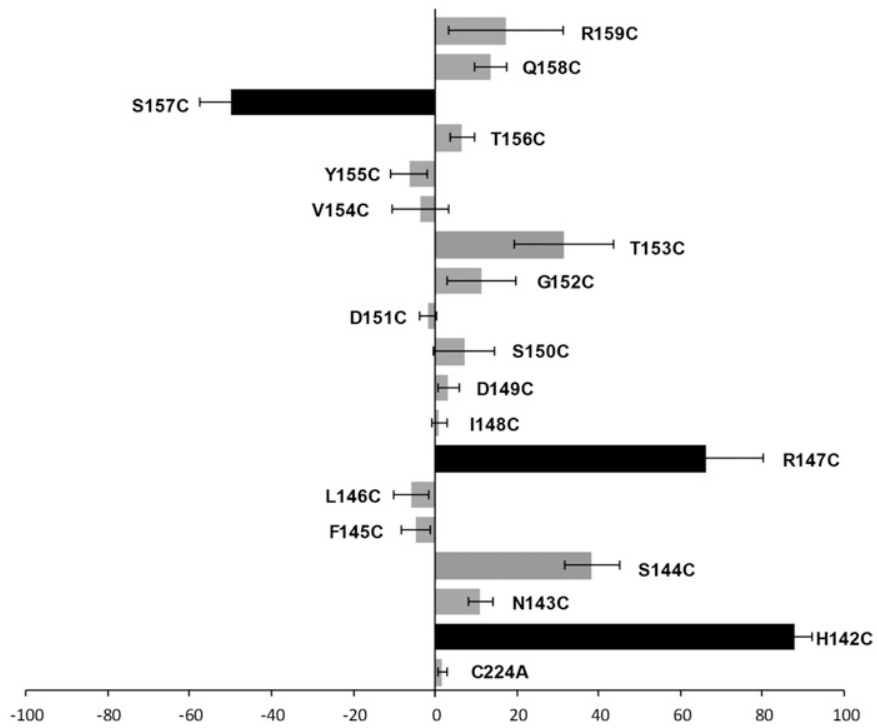

Fig. 4. Effect of MTSET on current evoked from GABA activation of HcoUNC-49BC receptors with mutated Hco-UNC49-B subunits. (A) Representative tracings of current elicited from $\mathrm{GABA}\left(\mathrm{EC}_{20-50}\right)$ before and after MTSET application (1 mM, 1 minute). (B) Percentage change in current from treatment of mutant Hco-UNC-49BC receptors with MTSET. Each bar indicates the mean \pm S.E. $(n>3)$. Black bars denote statistically significant difference from C224A $(P<0.001)$.
MD Simulations. As a further analysis of loop E residues we conducted molecular simulations. To ensure that the simulations provided a meaningful analysis of the role of loop $\mathrm{E}$ residues and their possible affect on GABA binding, we first examined the interaction of GABA with well-studied residues within the binding pocket. During the course of the simulation, the GABA molecule remained inside the initial binding pocket and interacted with key side chains previously implicated as essential for GABA binding in both mammalian (Newell et al., 2004) and the UNC-49 receptor (Accardi and Forrester, 2011; Kaji et al., 2015) (Fig. 6A). For example the positive amino group of GABA forms ionic bonds with carboxylate of E185 (loop B) $94 \%$ of the time. Likewise the carboxylate group of GABA forms ionic bonds with R87 (loop D) 99\% of the time, and S236 (loop C) forms hydrogen bonds with GABA 35\% of the time. These values were similar to MD simulations of the Drosophila resistance to dieldrin (RDL) receptor (Ashby et al., 2012).

With respect to loop $\mathrm{E}$ residues, the molecular simulations revealed only two residues forming hydrogen bonding with GABA. GABA most frequently formed H-bonds with S157 (74.25\% of the time) and TYR155 (53.36\% of the time) (Fig. 6, A and D).

ARG147 did not interact with the GABA molecule, except for during the first few nanoseconds of the simulation (Fig. 6B; Fig. 7A). Alternately, the R147 side chain mainly associated with the D149 side chain (Fig. 7A) via an ionic bond (35\%
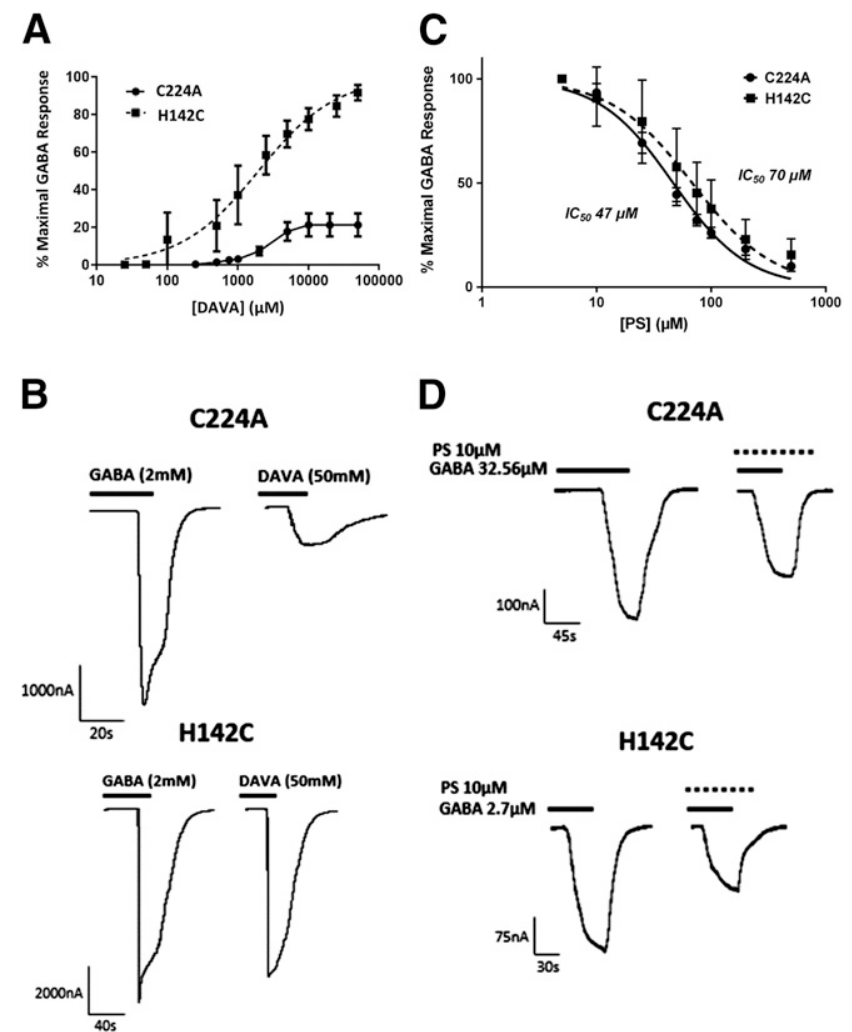

Fig. 5. Effect of loop E H142C mutation in Hco-UNC-49B on Hco-UNC49BC response to DAVA and PS. (A) DAVA dose-response curve comparing $\mathrm{C} 224 \mathrm{~A}$ and $\mathrm{H} 142 \mathrm{C}$ mutant receptors. (B) Comparison of maximal current produced by GABA and DAVA in C224A and H142C mutant receptors. (C) PS dose-response curve comparing C224A and $\mathrm{H} 142 \mathrm{C}$ mutant receptors. (D) Comparison of current inhibited by PS in the presence of $\mathrm{GABA}\left(\mathrm{EC}_{50}\right)$ in $\mathrm{C} 224 \mathrm{~A}$ and $\mathrm{H} 142 \mathrm{C}$ mutant receptors. 
TABLE 2

$\mathrm{EC}_{50}$, Hill coefficient, and maximal responses of DAVA and GABA produced by mutant Hco-UNC-49BC receptors H142C and C224A

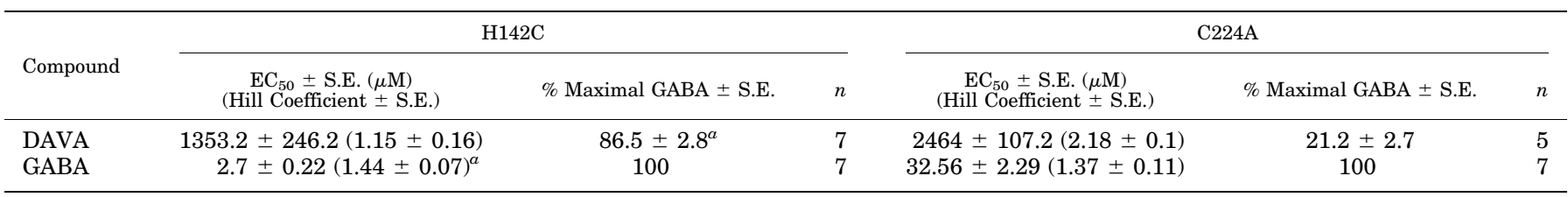

${ }^{a}$ Values statistically significantly different from $\mathrm{C} 224 \mathrm{~A}(P<0.001)$.

during the course of the simulation). However, the most recurrent interaction between the two residues was a $\mathrm{H}$-bond between the backbone of R147 and Y155 as part of the antiparallel $\beta$-sheet secondary structure of loop $\mathrm{E}(\sim 81 \%$ as an acceptor and $\sim 19 \%$ as a donor) (Fig. 6 B; Fig. 7B).

Electrostatic interactions between H142 and S144 were also observed. However, the interaction was variable, and only $0.39 \%$ of the time was the interaction hydrogen bonding. The distance between these two groups fluctuated between $\sim 1 \mathrm{~nm}$ (not interacting) and $\sim 0.4 \mathrm{~nm}$ (interacting) (Fig. 6C; Fig. 8). For the last 200 nanoseconds of the simulation, the separation distance became more dynamic. Further investigation indicated that there was a small structural change in the structure of loop E at $t \sim 300$ nanoseconds, where the S144 and F145 (at the beginning of the loop) lose their $\beta$-structure (Supplemental Fig. 1). However, this change did not have any major impact on the results calculated in the system (Supplemental Fig. 2; Supplemental Table 1).

\section{Discussion}

A comparative analysis of residues between mammalian and invertebrate GABA receptors can provide some valuable information on ligand-binding sites and receptor function. Indeed, a comparative mutagenesis approach was key to identifying the neurosteroid binding site in cys-loop GABA receptors (Hosie et al., 2006). Our study describes the characterization of the 18 residues found in binding loop $\mathrm{E}$ of the Hco-UNC-49 receptor.

In this investigation we determined which residues, when mutated to cysteine, affected the sensitivity of the Hco-UNC-49 receptor to GABA and other agonists. Moreover, exposure of the introduced cysteines to the reducing agent MTSET revealed which residues were accessible to MTSET modification (e.g., in an aqueous environment) and affected GABA sensitivity. However, the SCAM results should interpreted with caution because molecules such as MTSET have been shown to have more complex effects, such as acting as tethered partial agonists (Wang et al., 2010). Alternatively the enhancement in $I_{\mathrm{GABA}}$ might be caused by MTSET acting as a positive allosteric modulator.

This study revealed some loop E residues within Hco-UNC-49 that may exhibit similar properties as those found in human receptors, but it also revealed several with possible distinct functions in the nematode receptor. Overall, it appears that the loop $\mathrm{E}$ of the Hco-UNC-49 receptor is more tolerant to cysteine mutagenesis than the $\mathrm{GABA}_{\mathrm{A}}$ receptor (Kloda and Czajkowski, 2007).

Another distinction in the overall results of this study was that only one of the mutations (S157C) resulted in a decrease in $I_{\mathrm{GABA}}$ when modified by MTSET whereas others exhibited either no change or an increase in current. Studies on the human $\mathrm{GABA}_{\mathrm{C}}$ (which also used MTSET) receptor appeared to show the opposite trend (Sedelnikova et al., 2005). The results presented here may indicate an overall difference in the structure of loop $\mathrm{E}$ and the role it may play in nematode cys-loop GABA receptors when compared with their mammalian counterparts.
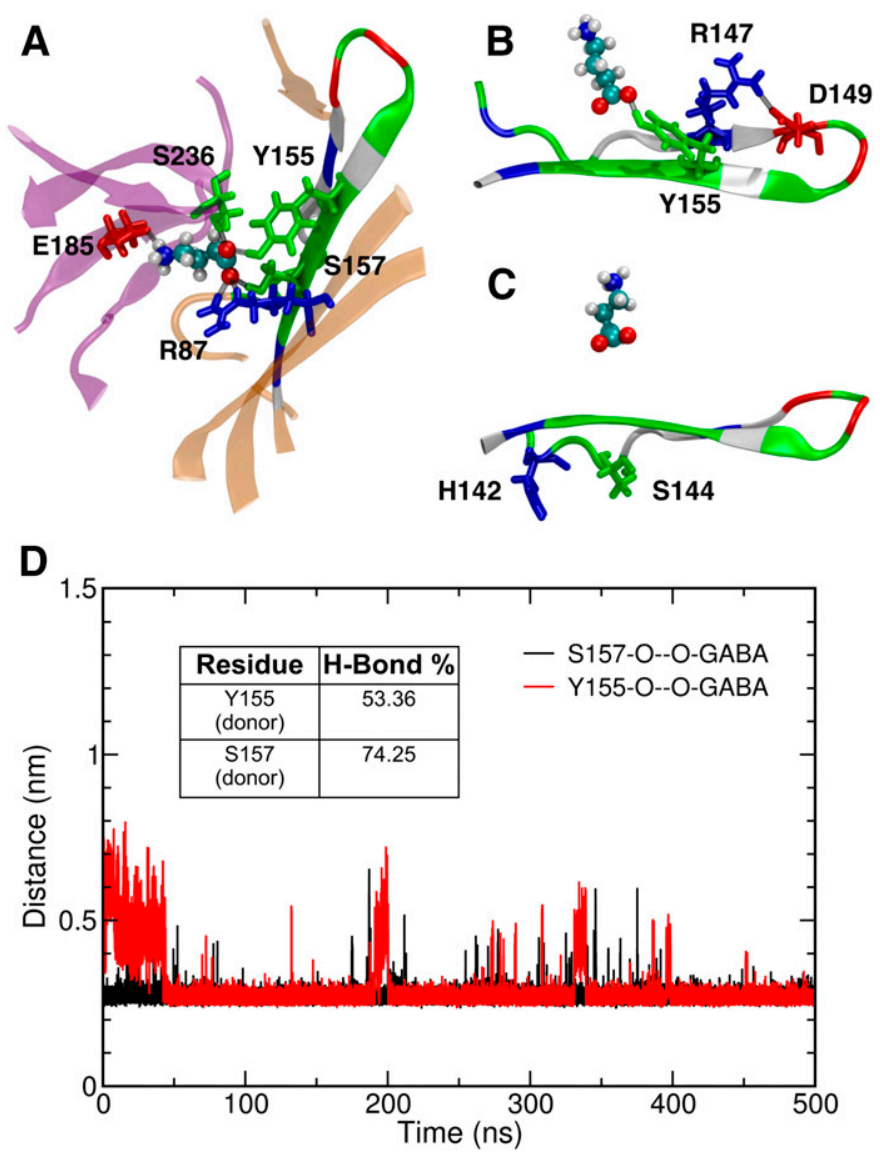

Fig. 6. Molecular simulation snapshots of the Hco-UNC-49B homodimer with GABA docked. E185 is in loop B, S236 is in loop C, R87 is in loop D, and H142, S144, R147, D149, Y155, and S157 are in loop E. (A) GABA interacting with nearby residues within the binding pocket. Gray dashed lines represent the H-bonds. The orange-colored backbone and displayed loop $\mathrm{E}$ are part of the same subunit; the purple-colored regions belong to the other subunit of the dimer. (B) Interaction between GABA, Y155, R147, and D149 and (C) H142 interacting with S144. Loop E is presented in residue type colors. The positively charged residues are in blue, the negatively charged residues are in red, the polar residues are in green, and nonpolar residues are in white. (D) Distance analysis between GABA and Y155 and S157 over the course of the simulation. The distances are calculated between the oxygens of the OH group on the side chains of Y155 and S157 and oxygens of GABA. Inset: Percentage of hydrogen bonding between residues and GABA. Residue numbering for Hco-UNC-49B corresponds to the first methionine of the amino acid sequence. PBD File: Hco-UNC-49. 

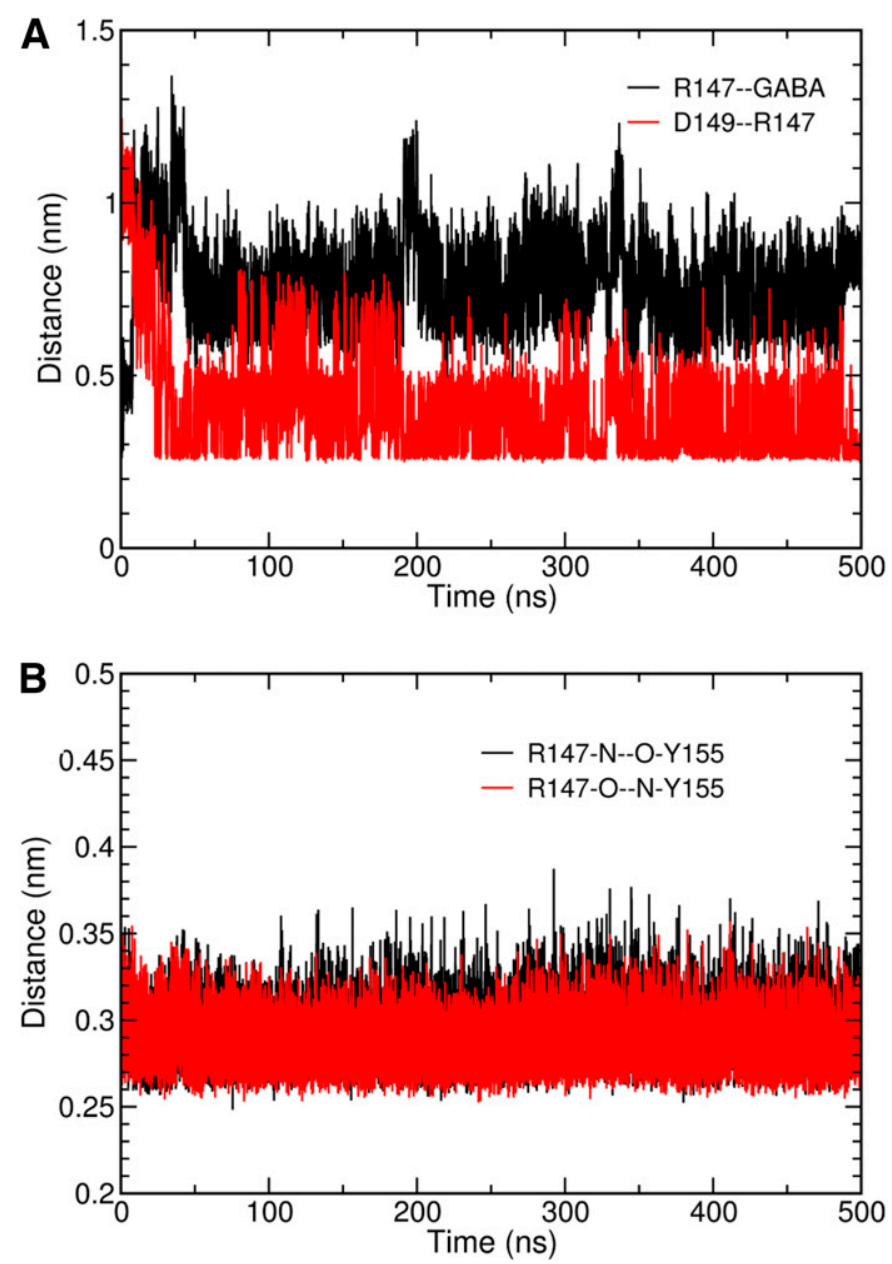

Fig. 7. MD analysis of the interactions between residues and GABA (A) The minimum distance between the three nitrogen atoms of R147 sidechain and the two oxygen atoms of the GABA (black), and the oxygen atom of the D149 side chain and the three nitrogen atoms of the R147 side chain (red). The black line indicates an initial interaction between the R147 and the GABA molecule and separation afterward; the red line indicates an initial separation between the R147 and the D149 and interaction for the rest of the simulation. (B) Minimum distances between the oxygen and nitrogen atoms in the backbone of the R147 and the Y155.

In this study six mutants (I148C, D149C, S150C, D151C, G152C, and Q158C) did not affect the functionality of the receptor, exhibiting $\mathrm{EC}_{50}$ values close to those obtained from C224A (the cysteine-less mutant displaying WT function). According to our model (Fig. 2), I148 to G152 are at the top of loop $\mathrm{E}$ and therefore positioned $13-17 \AA$ away from GABA. Interestingly, the UNC-49 G152 residue, which is conserved among all GABA receptors, appears to be much more tolerant to a change to cysteine (1.7-fold decrease), compared with mammalian receptors where this change resulted in either a 42-fold reduction in GABA sensitivity or nonfunctional receptors (Sedelnikova et al., 2005; Kloda and Czajkowski, 2007).

R147 appears to be conserved across many phyla (Fig. 2). We found a small 2.7-fold change in $\mathrm{EC}_{50}$ when this arginine was mutated to a cysteine; when MTSET was applied, there was a significant increase in GABA current. These results are very different from what was found for the $\mathrm{GABA}_{\mathrm{A}} \alpha 1$ and the $\mathrm{GABA}_{\mathrm{C}} \rho 1$ receptors. When this arginine residue was mutated

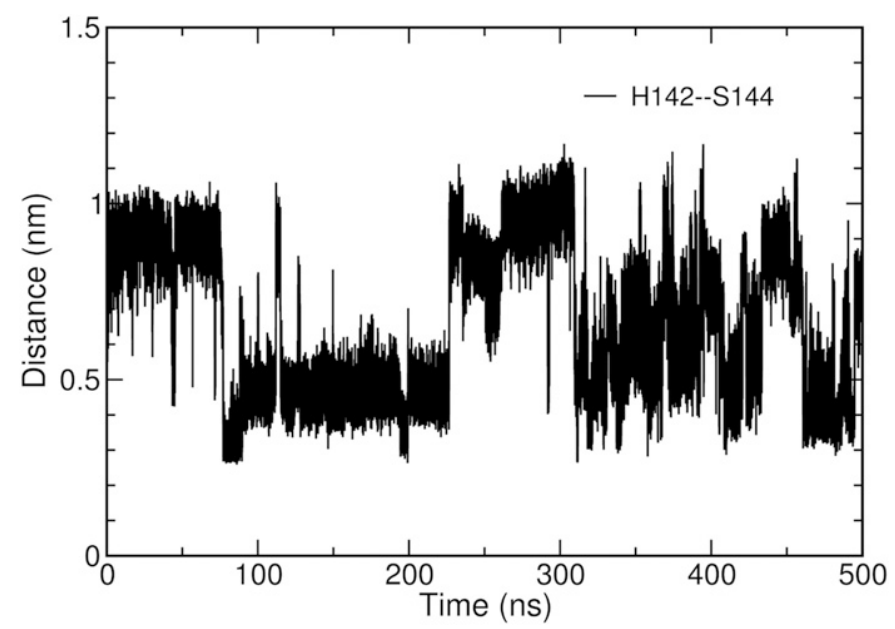

Fig. 8. The minimum distance between the two nitrogen atoms of H142 sidechain and the oxygen atom of the S144 side chain.

to a cysteine in the $\mathrm{GABA}_{\mathrm{A}} \alpha 1$ subunit, there was a 260 -fold decrease in $\mathrm{EC}_{50}$ compared with that of the WT (Kloda and Czajkowski, 2007), and the same mutation in GABAc $\rho 1$ resulted in a receptor that was expressed too low for further analysis (Sedelnikova et al., 2005). However, in both receptors it was determined that this arginine does not directly interact with GABA.

Homology modeling of the Hco-UNC-49 receptor suggests that R147 faces the binding pocket but does not interact with GABA, based on our MD simulations. However, the positive charge of MTSET (when bound to the introduced cysteine) may stabilize the negative carboxyl group of GABA, which may explain the increase in GABA current we observed after application. In the insect RDL receptor, mutations at this position (i.e., R166) with either an A or G produced nonfunctional channels. However, it has been suggested that this residue was not likely to be essential for GABA binding (Ashby et al., 2012).

It is possible that $\mathrm{R} 147$ has a different role in nematode UNC-49 receptors compared with mammalian and possibly even Drosophila receptors. We found evidence for a molecular interaction between the side chains of R147 and D149 (Fig. 7A). In the $\mathrm{GABA}_{\mathrm{A}} \alpha 1$ and the $\mathrm{GABA}_{\mathrm{C}} \rho 1$ receptors the residues in the analogous positions as D149 are nonionic polar residues (Fig. 2). Therefore, the potential interactions between $\mathrm{R} 147$ and other residues may provide some unique features of the nematode GABA receptor.

H142C produced a hypersensitive channel. Treatment with MTSET revealed that the introduced cysteine was accessible for modification, and the resulting modification increased the GABA current. Our MD simulations suggests that H142 does not face the binding pocket, so it may affect the binding site in other ways. Indeed, the mutation $\mathrm{H} 142 \mathrm{C}$ not only had a positive effect on GABA, but also affected the Hco-UNC-49 receptor partial agonist DAVA, converting it to a full agonist. This mutation had minimal effect on the sensitivity of the neurosteroid PS, a negative allosteric modulator shown to bind at a site away from the agonist binding site (Wardell et al., 2006). Because histidine is an amino acid with a positively charged bulky side group, it is possible that its removal results in structural changes that may positively 
affect both GABA and DAVA binding. One possibility is that H142 has some electrostatic interaction with S144 (Fig. 6C; Fig. 8) and the H142C change alters this interaction, which in turn alters the binding site. It is interesting that $\mathrm{H} 142$ appears to be conserved within the nematode phyla and is also present in the unique nematode cation GABA receptor EXP-1 (Beg and Jorgensen, 2003). The exact role of this residue in nematode receptors is unknown, but it could be involved in the unique pharmacologic properties of nematode UNC-49-like GABA receptors.

One residue that may have similar functions across phyla is S157. Mutation of this position to cysteine produces a receptor that was 113-fold less sensitive to GABA; when S157C is modified by MTSET, the GABA current is reduced. The analogous residues in human GABA receptors are T129 in the $\mathrm{GABA}_{\mathrm{A}}$ receptor and $\mathrm{S} 168$ in the $\mathrm{GABA}_{\mathrm{C}}$ receptor. In both cases these residues were confirmed to lie within the binding pocket (Sedelnikova et al., 2005; Kloda and Czajkowski, 2007) where they form an H-bond with GABA. The molecular simulations also revealed high occurrences of $\mathrm{H}$-bonds between S157 and GABA. In the insect RDL receptor it appears that a serine at this position is also essential, where it makes a H-bond with the carboxyl group of GABA (Ashby et al., 2012). However, in the Hco-UNC-49 receptor, MD simulations also detected less frequent H-bonding between Y155 and GABA. The equivalent residue in mammalian receptors and several other GABA receptors is a nonpolar leucine. We anticipate, that in the Hco-UNC-49 receptor, Y155 may play a minor role in GABA binding. Whether it contributes to some unique functions of the nematode UNC-49 receptor will be focus of further study.

\section{Conclusion}

This was the first study to conduct a comprehensive analysis of a single binding loop from an invertebrate GABA receptor. We found that, of the loop $\mathrm{E}$ mutants we analyzed, H142C, R147C, and S157C had significant changes in $\mathrm{EC}_{50}$ and were accessible to modification by MTSET, which affected GABA sensitivity. Furthermore, as a result of this study, H142 was identified as a residue that may have an important role in the sensitivity and selectively of the UNC-49 receptor. More work is required to fully characterize the structure of the Hco-UNC-49 binding pocket, but the present study suggests that there are structural differences when compared with mammalian receptors. This further highlights the potential of Hco-UNC-49 as a future drug target.

\section{Acknowledgments}

We thank Micah Callanan for assistance with the molecular modeling.

\section{Authorship Contributions}

Participated in research design: Kwaka, de Haan, Forrester. Conducted experiments: Kwaka, Hassan Khatami, Foster, Cochrane, Habibi.

Contributed new reagents or analytic tools: de Haan, Forrester. Performed data analysis: Kwaka, Hassan Khatami.

Wrote or contributed to the writing of the manuscript: Kwaka, Hassan Khatami, Forrester.

\section{References}

Abdelmassih SA, Cochrane E, and Forrester SG (2017) Evaluating the longevity of surgically extracted Xenopus laevis oocytes for the study of nematode ligand-gated ion channels. Invert Neurosci 18:1.

Accardi MV and Forrester SG (2011) The Haemonchus contortus UNC-49B subunit possesses the residues required for GABA sensitivity in homomeric and heteromeric channels. Mol Biochem Parasitol 178:15-22.

Ashby JA, McGonigle IV, Price KL, Cohen N, Comitani F, Dougherty DA, Molteni C, and Lummis SC (2012) GABA binding to an insect GABA receptor: a molecular dynamics and mutagenesis study. Biophys $J$ 103:2071-2081.

Bamber BA, Beg AA, Twyman RE, and Jorgensen EM (1999) The Caenorhabditis elegans unc- 49 locus encodes multiple subunits of a heteromultimeric GABA receptor. J Neurosci 19:5348-5359.

Bamber BA, Richmond JE, Otto JF, and Jorgensen EM (2005) The composition of the GABA receptor at the Caenorhabditis elegans neuromuscular junction. $\mathrm{Br} J$ Pharmacol 144:502-509.

Bamber BA, Twyman RE, and Jorgensen EM (2003) Pharmacological characterization of the homomeric and heteromeric UNC-49 GABA receptors in C. elegans. $\mathrm{Br} J$ Pharmacol 138:883-893.

Bartos M, Corradi J, and Bouzat C (2009) Structural basis of activation of cys-loop receptors: the extracellular-transmembrane interface as a coupling region. $\mathrm{Mol}$ Neurobiol 40:236-252.

Beg AA and Jorgensen EM (2003) EXP-1 is an excitatory GABA-gated cation channel. Nat Neurosci 6:1145-1152.

Best RB, Zhu X, Shim J, Lopes PEM, Mittal J, Feig M, and Mackerell AD Jr (2012) Optimization of the additive CHARMM all-atom protein force field targeting improved sampling of the backbone $\phi, \psi$ and side-chain $\chi(1)$ and $\chi(2)$ dihedral angles. $J$ Chem Theory Comput 8:3257-3273.

Brejc K, van Dijk WJ, Klaassen RV, Schuurmans M, van Der Oost J, Smit AB, and Sixma TK (2001) Crystal structure of an ACh-binding protein reveals the ligand-binding domain of nicotinic receptors. Nature 411:269-276.

del Olmo N, Bustamante J, del Río RM, and Solís JM (2000) Taurine activates GABA (A) but not $\mathrm{GABA}(\mathrm{B})$ receptors in rat hippocampal CA1 area. Brain Res 864: 298-307.

Hibbs RE and Gouaux E (2011) Principles of activation and permeation in an anionselective Cys-loop receptor. Nature 474:54-60.

Hosie AM, Aronstein K, Sattelle DB, and ffrench-Constant RH (1997) Molecular biology of insect neuronal GABA receptors. Trends Neurosci 20:578-583.

Hosie AM, Wilkins ME, da Silva HM, and Smart TG (2006) Endogenous neurosteroids regulate $\mathrm{GABA}_{\mathrm{A}}$ receptors through two discrete transmembrane sites. Nature 444:486-489.

Humphrey W, Dalke A, and Schulten K (1996) VMD: visual molecular dynamics. $J$ Mol Graph 14:33-38, 27-28 http://www.ks.uiuc.edu/Research/vmd/.

Irwin JJ, Sterling T, Mysinger MM, Bolstad ES, and Coleman RG (2012) ZINC: a free tool to discover chemistry for biology. $J$ Chem Inf Model 52:1757-1768.

Jones AK and Sattelle DB (2008) The cys-loop ligand-gated ion channel gene superfamily of the nematode, Caenorhabditis elegans. Invert Neurosci 8:41-47.

Kaji MD, Kwaka A, Callanan MK, Nusrat H, Desaulniers JP, and Forrester SG (2015) A molecular characterization of the agonist binding site of a nematode cysloop GABA receptor. $\mathrm{Br} J$ Pharmacol 172:3737-3747.

Kloda JH and Czajkowski C (2007) Agonist-, antagonist-, and benzodiazepineinduced structural changes in the $\alpha 1 \mathrm{Met}^{113}-\mathrm{Leu}^{132}$ region of the $\mathrm{GABA}_{\mathrm{A}}$ receptor Mol Pharmacol 71:483-493.

Kusama T, Spivak CE, Whiting P, Dawson VL, Schaeffer JC, and Uhl GR (1993) Pharmacology of GABA rho 1 and GABA alpha/beta receptors expressed in Xenopus oocytes and COS cells. Br J Pharmacol 109:200-206.

Laing R, Kikuchi T, Martinelli A, Tsai IJ, Beech RN, Redman E, Holroyd N, Bartley DJ, Beasley H, Britton C, et al. (2013) The genome and transcriptome of Haemonchus contortus, a key model parasite for drug and vaccine discovery. Genome Biol 14(8): R88

Morris GM, Huey R, Lindstrom W, Sanner MF, Belew RK, Goodsell DS, and Olson AJ (2009) AutoDock4 and AutoDockTools4: automated docking with selective receptor flexibility. J Comput Chem 30:2785-2791.

Newell JG, McDevitt RA, and Czajkowski C (2004) Mutation of glutamate 155 of the GABAA receptor beta2 subunit produces a spontaneously open channel: a trigger for channel activation. $J$ Neurosci 24(50):11226-11235.

Putrenko I, Zakikhani M, and Dent JA (2005) A family of acetylcholine-gated chloride channel subunits in Caenorhabditis elegans. $J$ Biol Chem 280:6392-6398.

Sali A and Blundell TL (1993) Comparative protein modelling by satisfaction of spatial restraints. J Mol Biol 234:779-815.

Sedelnikova A, Smith CD, Zakharkin SO, Davis D, Weiss DS, and Chang Y (2005 Mapping the rho1 GABA $(\mathrm{C})$ receptor agonist binding pocket. Constructing a complete model. J Biol Chem 280:1535-1542.

Siddiqui SZ, Brown DDR, Rao VTS, and Forrester SG (2010) An UNC-49 GABA receptor subunit from the parasitic nematode Haemonchus contortus is associated with enhanced GABA sensitivity in nematode heteromeric channels. $J$ Neurochem 113:1113-1122.

Trott O and Olson AJ (2010) AutoDock Vina: improving the speed and accuracy of docking with a new scoring function, efficient optimization, and multithreading. $J$ Comput Chem 31:455-461.

Vanommeslaeghe K, Hatcher E, Acharya C, Kundu S, Zhong S, Shim J, Darian E, Guvench O, Lopes P, Vorobyov I, et al. (2010) CHARMM general force field: a force field for drug-like molecules compatible with the CHARMM all-atom additive biological force fields. J Comput Chem 31:671-690.

Vanommeslaeghe K and MacKerell AD Jr (2012) Automation of the CHARMM General Force Field (CGenFF) I: bond perception and atom typing. $J$ Chem Inf Model 52:3144-3154

Vanommeslaeghe K, Raman EP, and MacKerell AD Jr (2012) Automation of the CHARMM General Force Field (CGenFF) II: assignment of bonded parameters and partial atomic charges. J Chem Inf Model 52:3155-3168. 
Wang J, Horenstein NA, Stokes C, and Papke RL (2010) Tethered agonist analogs as site-specific probes for domains of the human $\alpha 7$ nicotinic acetylcholine receptor that differentially regulate activation and desensitization. Mol Pharmacol 78: $1012-1025$.

Wardell B, Marik PS, Piper D, Rutar T, Jorgensen EM, and Bamber BA (2006) Residues in the first transmembrane domain of the Caenorhabditis elegans GABA (A) receptor confer sensitivity to the neurosteroid pregnenolone sulfate. $\mathrm{Br} J$ Pharmacol 148:162-172.

Woodward RM, Polenzani L, and Miledi R (1993) Characterization of bicuculline/baclofen-insensitive (rho-like) gamma-aminobutyric acid receptors expressed in Xenopus oocytes. II. Pharmacology of gamma-aminobutyric acidA and gamma-aminobutyric acidB receptor agonists and antagonists. Mol Pharmacol 43: $609-625$.

Yu W, He X, Vanommeslaeghe K, and MacKerell AD Jr (2012) Extension of the CHARMM General Force Field to sulfonyl-containing compounds and its utility in biomolecular simulations. J Comput Chem 33:2451-2468.

Address correspondence to: Dr. Sean G. Forrester, University of Ontario Institute of Technology, 2000 Simcoe Street North, Oshawa, ON L1H 7K4, Canada. E-mail: sean.forrester@uoit.ca 